\title{
Affective and cognitive empathy in adolescents with autism spectrum disorder
}

\section{Monica Mazza ${ }^{1}$ *, Maria C. Pino ${ }^{1}$, Melania Mariano ${ }^{1}$, Daniela Tempesta ${ }^{1}$, Michele Ferrara $^{1}$, Domenico De Berardis ${ }^{2}$, Francesco Masedu ${ }^{3}$ and Marco Valenti ${ }^{3,4}$}

${ }^{1}$ Department of Life, Health and Environmental Sciences, University of L'Aquila, L'Aquila, Italy

2 Psychiatric Service of Diagnosis and Treatment, Department of Mental Health, G. Mazzini Hospital, Teramo, Italy

${ }^{3}$ Department of Applied Clinical Sciences and Biotechnology, Section of Clinical Epidemiology and Environmental Medicine, University of L'Aquila, L'Aquila, Italy

${ }^{4}$ Reference Regional Centre for Autism, Abruzzo Region Health System, L'Aquila, Italy

\section{Edited by:}

Srikantan S. Nagarajan, University of California, San Francisco, USA

\section{Reviewed by:}

Simon Baron-Cohen, University of Cambridge, UK

Peter Scott Pressman, University of California, San Francisco, USA

\section{*Correspondence:}

Monica Mazza, Department of Life, Health and Environmental Sciences, University of L'Aquila, Via Vetoio, Località Coppito, 67100 L'Aquila, $A Q$, Italy

e-mail:monica.mazza@cc.univaq.it
The broad construct of empathy incorporates both cognitive and affective dimensions. Recent evidence suggests that the subjects with autistic spectrum disorder (ASD) show a significant impairment in empathic ability. The aim of this study was to evaluate the cognitive and affective components of empathy in adolescents with ASD compared to controls. Fifteen adolescents with ASD and 15 controls underwent paper and pencil measures and a computerized Multifaceted Empathy Test. All measures were divided into mentalizing and experience sharing abilities. Adolescents with ASD compared to controls showed deficits in all mentalizing measures: they were incapable of interpreting and understanding the mental and emotional states of other people. Instead, in the sharing experience measures, the adolescents with ASD were able to empathize with the emotional experience of other people when they express emotions with positive valence, but were not able to do so when the emotional valence is negative. These results were confirmed by the computerized task. In conclusion, our results suggest that adolescents with ASD show a difficulty in cognitive empathy, whereas the deficit in affective empathy is specific for the negative emotional valence.

Keywords: adolescents, autistic spectrum disorder (ASD), affective empathy, cognitive empathy, experience sharing, mentalizing

\section{INTRODUCTION}

Autistic Spectrum Disorder (ASD) is a triad of qualitative impairments in social interaction, communication and restricted, repetitive, and stereotyped behaviors (American Psychiatric Association, 2000). An important feature of the proposed criteria in DSM-5 for ASD is a change from three (autistic triad) to two domains: "social/communication deficits" and "fixated and repetitive pattern of behavior" (Wilkinson, 2012). These difficulties often make it very hard for people with ASD to be successful members of society and can present very serious challenges to parents, teachers, and other professionals. Major difficulties in social interaction have been a defining feature of individuals with autism (Fletcher-Watson et al., 2014).

People with ASD often show an impaired comprehension of other people's mental states, such as thoughts, beliefs, and intentions (Frith and Happé, 1994; Frith and Frith, 2003; Jones et al., 2010; Gaigg, 2012; Schwenck etal., 2012). Recent studies showed that subjects with ASD have not only a difficulty in attributing another person's mental state but also in the capacity to respond to another person's mental state with an appropriate emotion (Sucksmith etal., 2013). These abilities seem to be involved in the multifaceted construct of empathy (Zaki and Ochsner, 2012). In agreement with recent literature (Jones etal., 2010; Baron-Cohen, 2011; Dziobek etal., 2011; Schwenck et al., 2012; Zaki and Ochsner, 2012) empathy should no longer be considered as a unitary concept, instead it comprises at least two components (Singer, 2006; Decety and Meyer, 2008; Dziobek et al., 2008). In fact, empathy includes the ability to understand what others are thinking or feeling, without necessarily "resonating" with that feeling state (cognitive empathy) and the ability to emotionally "resonate" with other people's feelings while understanding that they are distinct from one's own (affective empathy; Jones et al., 2010; Schwenck et al., 2012). The cognitive dimension of empathy requires complex cognitive functions, including perspective-taking and mentalizing (ShamayTsoory et al., 2002, 2009; Shamay-Tsoory, 2011; Zaki and Ochsner, 2012), whereas affective empathy includes experience sharing of other persons' internal states (Zaki and Ochsner, 2012). Emotional contagion is a precursor of affective empathy, whereby embodiment entails the forming of a representation of the other person's feelings, and thereby sharing of their experience (Hadjikhani et al., 2014).

Thus, mentalizing and experience sharing apparently represent two aspects of the same object, i.e., understanding and responding to another person's internal states, involving different mental systems. Mentalizing ability examines the theory of mind (ToM) capacity by asking subjects to draw explicit inferences about the mental states of other people. Experience sharing is the tendency to take on, resonate with, or "share" the emotions of others and it is often tied to a mechanism known as "internal resonance" (Zaki and Ochsner, 2012). 
It is widely accepted that subjects with ASD do not possess a fully functioning ToM; even high functioning adults with ASD may struggle with complex ToM tasks (Ponnet et al., 2004; Fletcher-Watson etal., 2014). However, affective impairments found in people with ASD are mainly related to the cognitive recognition and processing of emotions, rather than to the actual ability to feel emotional distress or concern. The lack of a clear distinction between affective and cognitive empathy has led to an incomplete understanding of the empathic abilities of individuals with ASD. Interestingly, a few studies have formally assessed empathy in individuals with autistic conditions (Dziobek etal., 2008; Jones etal., 2010; Schwenck etal., 2012). Dziobek etal. (2008) showed an impairment in cognitive empathy, but the presence of normal empathetic concern (affective empathy) in adults with Asperger syndrome (AS), based on self-report questionnaires such as the Interpersonal Reactivity Index (Davis, 1980; Rogers etal., 2007) and the Multifaceted Empathy Test (MET; Dziobek et al., 2008).

Moreover, another two studies (Jones et al., 2010; Schwenck etal., 2012) using only paper and pencil measures, have confirmed that ASD is characterized by difficulties in mentalizing ability (cognitive empathy), but not with affective empathy (Lockwood et al., 2013).

The study of social skills in adolescents with ASD is crucial also for the construction of rehabilitation paradigms to improve empathic capacities. For this reason, in this study we investigated the empathic ability in adolescents with ASD compared to controls, using both paper and pencil and computerized measures, divided into mentalizing and experience sharing abilities in accordance with Zaki and Ochsner's (2012) model, to evaluate the presence of a dissociation between cognitive and affective empathic abilities in this population.

\section{MATERIALS AND METHODS}

The study included 30 participants: 15 adolescents (11 boys and 4 girls, mean age \pm SD: $15.11 \pm 4.89$ years) were affected by ASD and 15 control subjects ( 10 boys and 5 girls; mean age $=16.50 \pm 6.23$ ), were recruited to match the ASD group with respect to age and education.

Autistic spectrum disorder participants were selected by the Reference Regional Centre for Autism, Abruzzo Region Health System, L'Aquila (Italy). The ASD diagnosis were given by experienced clinicians according to the new criteria of the DSM-5 (American Psychiatric Association, 2013). ASD diagnosis of patients was made with Autism Diagnostic Observation Schedule, Second Edition (ADOS-2; Lord et al., 2012).

Socio-demographic and clinical information of all the participants are summarized in Table 1. The parents of adolescents provided informed consent to participate in the study.

\section{MENTALIZING MEASURES \\ First-order false belief test}

This task was designed to elicit a response that demonstrated the ability to make inferences about another individual's mental state, namely, that a character in the story holds a false belief. First order false beliefs require a subject to make an inference about the state of the world. To assess first order ToM two stories were used: The washing machine task (Rowe et al., 2001; Mazza et al., 2007) and the Cigarettes Task (Happé, 1994).

Each subject obtained a score ranging from 0 to 1 for each question. If the subject gave a correct answer to both the first order stories, s/he had a global score for first order ToM equal to 2 (non-casual performance).

\section{Advanced Theory of Mind Task}

This task is an Italian adaptation of a cognitive task used by Blair and Cipolotti (2000) and proposed in the literature by Happé

Table 1 | Socio-demographic, mentalizing, and sharing experience measure.

\begin{tabular}{|c|c|c|c|c|}
\hline & $\begin{array}{l}\text { ASD }(n=15) \\
\text { mean scores (SD) }\end{array}$ & $\begin{array}{l}\text { Controls ( } n=15 \text { ) } \\
\text { means scores (SD) }\end{array}$ & $t(d f=28)$ & $p$ \\
\hline Age (years) & $15.11(4.89)$ & $16.50(6.23)$ & -0.686 & 0.498 \\
\hline Gender & $11 \mathrm{M}, 4 \mathrm{~F}$ & $10 \mathrm{M}, 5 \mathrm{~F}$ & & \\
\hline Education (years) & $10.45(2.55)$ & $10.83(1.85)$ & -0.668 & 0.507 \\
\hline Raven's matrices (in percentiles) & $57.27(26.96)$ & $43.57(22.30)$ & 1.119 & 0.279 \\
\hline \multicolumn{5}{|l|}{ Mentalizing measures } \\
\hline Advanced ToM task & $6.69(4.15)$ & $12.33(0.77)$ & -4.626 & 0.0001 \\
\hline BES cognitive subscale & $29.27(4.01)$ & $39.83(6.57)$ & -5.154 & 0.0001 \\
\hline \multicolumn{5}{|l|}{ Sharing experience measures } \\
\hline Emotion attribution task & $27.41(11.67)$ & $46.75(10.21)$ & -4.618 & 0.001 \\
\hline Positive emotions & $7.57(2.92)$ & $9.42(0.51)$ & -2.149 & 0.068 \\
\hline Negative emotions & 6.24 (1.59) & $8.25(1.81)$ & -2.803 & 0.011 \\
\hline Eyes Task & $14.50(8.25)$ & $29.92(8.62)$ & -3.142 & 0.004 \\
\hline BES-affective subscale & $30(3.46)$ & $32.17(7.38)$ & -7.38 & 0.322 \\
\hline
\end{tabular}

Mean scores (and SDs) to the psychological tests separately for ASD and control subjects. The significant results are highlighted by bold numbers. 
(1994). The task consists of a short version of 13 vignettes, each accompanied by two questions; the comprehension question "Was it true, what X said?," and the justification question "Why did X say that?" The 13 story-types included Lie, White Lie, Joke, Pretend, Misunderstanding, Double Bluff, and Contrary Emotion. Each subject obtained a score ranging from 0 to 1 for each question. The maximum score is 13 .

\section{Basic Empathy Scale-Cognitive Subscale}

The Basic Empathy Scale (BES) comprises a total of 20 items (Jolliffe and Farrington, 2006; Albiero et al., 2009). The cognitive empathy subscale (CE subscale, nine items), measures the ability to understand another person's emotions. Each item (e.g., "I can often understand how people are feeling even before they tell me") asks participants to express their own degree of agreement on a 5-point, Likert-type scale, ranging from 1 ("strongly disagree") to 5 ("strongly agree"). The BES has demonstrated a good validity (Jolliffe and Farrington, 2006; Albiero et al., 2009). Cronbach's a coefficient was calculated to examine the internal consistency of the scale, considered globally and in its two dimensions, as yielded by the confirmatory factor analysis. The results showed satisfactory internal consistency for both the scale and its subscales, given that the global scale $\alpha$ coefficient was 0.87 and cognitive subscale $\alpha$ values was 0.74 (Albiero et al., 2009).

\section{EXPERIENCE SHARING MEASURES}

The Eyes Task is a revised version of the "Reading the Mind in the Eyes Test" (Baron-Cohen et al., 2001). In brief, participants are given 36 photographs depicting the ocular area in an equal number of different actors and actresses. At each corner of every photo, four complex mental state descriptors, e.g., dispirited, bored, are printed, only one of which (the target word) correctly identifies the depicted person's mental state, while the others are included as foils. The test is scored by totaling the number of items (photographs) correctly identified by the participant; therefore, the maximum total score is 36. In the Italian version the internal consistency (Cronbach's alpha) was 0.605 . Test-retest reliability for the Eyes test, as measured by intraclass correlation coefficient, was 0.833 (95\% confidence interval $=0.745-0.902$ ).

The study of Vellante et al. (2013) confirms the validity of the Eyes test. Both internal consistency and test-retest stability were good for the Italian version of the Eyes test.

In the Emotion Attribution Task (Blair and Cipolotti, 2000). This task assessed ability to represent the emotions of others. In this task, the participant was presented with 58 short stories describing an emotional situation and was required to provide an emotion describing how the main character might feel in that situation. The sentences were designed to elicit attributions of positive and negative emotions. The task was scored according to the number of correct attributions. For this test as well, validation studies are lacking (Mazza et al., 2007).

The Basic Empathy Scale-Affective subscale (AE subscale, 11 items): measuring emotional congruence with another person's emotions. Example items included "I get caught up in other people's feelings easily." Each item asks participants to express their own degree of agreement on a 5-point, Likert-type scale, ranging from 1 ("strongly disagree") to 5 ("strongly agree"). Cronbach's alpha was 0.86 (Albiero et al., 2009).

\section{COGNITIVE AND AFFECTIVE EMPATHY MEASURES Multifaceted Empathy Test}

To assess empathy multi-dimensionally, we administered the MET (Dziobek et al., 2008), a measure of empathy that allows separate assessments of cognitive and affective aspects of empathic functioning. This test consists of a series of photographs that depict people in emotionally charged situations. In these pictures, taken from the International Affective Picture System (IAPS; Lang, 1980), the stimuli show individuals feeling different emotions: positive emotions ( 25 pictures that include emotions such as happiness, positive surprise), negative emotions (25 pictures that include emotions such as sadness, anger, disappointment).

Positive and negative emotions were presented in random order. All the stimuli were displayed on a black screen. For each picture the subjects were required to infer the emotional states of the individuals shown in the image by selecting one of four emotional state descriptors (cognitive empathy). To assess affective empathy, subjects rate their level of empathic concern for the individuals displayed in the images on a 9-point Likert scale.

\section{STATISTICAL METHODS}

Mann-Whitney $U$ test was used to analyse the level of significance of participants' scores on First-order false belief task.

$T$-test analysis was used to test significant differences between groups (ASD and control group) in socio-demographic, mentalizing (Advanced ToM and BES-cognitive subscale) and experience sharing measures (Eyes Task, emotion attribution task, and BES-affective subscale).

To evaluate the difference in MET performance between two groups, a $2 \times 4$ repeated measure design was used. The assumption of normality of the outcome variable was assessed carrying out a Kolmogorov-Smirnov non-parametric test. Restricted maximum likelihood estimation (REML) and an unstructured correlation have been used. Marginal effects have been calculated to getting an estimation of the way the presence of ASD affects the scores of each model predictor. The overall statistical significance of the model has been set at 0.05 level.

The Statistical Package for the Social Sciences (SPSS) software (version 22; SPSS Inc, Chicago, IL, USA) was used for calculating these statistics.

\section{RESULTS \\ MENTALIZING MEASURES}

The ASD group showed lower scores compared to the controls in the Advanced ToM Task $\left(T_{1,28}=-4.626 ; p=0.0001\right)$, and in BES cognitive subscale $\left(T_{1,28}=-5.154 ; p=0.0001\right)$.

In the First-order false belief task the groups differed significantly on the non-parametric Mann-Whitney $U$ test $(U=86.5$; $Z=-5.44 ; p=0.0001)$. The percentage of correct scores in Washing Machine and Cigarette Task was 25.8 and 45.2\% for ASD, whereas 100 and $80.8 \%$ for the controls, respectively.

Mentalizing performance scores (means and SD) are reported in Table 1. 


\section{EXPERIENCE SHARING MEASURES}

Adolescents with ASD showed lower scores compared to the control group in the Emotion Attribution Task total score $\left(T_{1,28}=-4.618 ; p=0.001\right)$, with a significant difference in negative emotions $\left(T_{1,28}=-2.803 ; p=0.011\right)$, but not in positive emotions $\left(T_{1,28}=-2.149 ; p=0.068\right)$. Adolescents with ASD also showed lower scores compared to the control group in the Eyes Task $\left(T_{1,28}=-3.142 ; p=0.004\right)$, but no significant differences in BES affective subscale $\left(T_{1,28}=-7.38 ; p=0.322\right)$ were found.

Experience sharing performance scores (means and SD) are reported in Table 1.

\section{AFFECTIVE AND COGNITIVE EMPATHY MEASURES Multifaceted Empathy Test}

Normalized MET data were analyzed by a linear mixed model for repeated measure design with REML. Analysis showed a significant group effect $(z=-1.18 \pm 0.18 ; p<0.05)$.

Marginal effects analysis showed statistically significant interaction between ASD and positive cognitive empathy (marginal effect $=-0.72 \pm 0.18 ; p<0.05)$ and between ASD and negative cognitive empathy (marginal effect $=-072 \pm 0.19 ; p<0.05$ ).

Results showed no significant difference between groups in positive affective empathy (marginal effect $=-0.18 \pm 0.21$; $p=0.519)$. On the other hand, ASD and controls differed in negative affective empathy (marginal effect $=-0.69 \pm 0.22 ; p<0.05$; see Table 2; Figure 1).

\section{DISCUSSION}

The aim of this study was to investigate the empathy dimensions in a sample of adolescents with ASD. Specifically, in our research, we examined the empathic abilities in an ASD group compared

Table 2 | Marginal effects of the linear mixed model for repeated measure design with restricted maximum likelihood estimation (REML) of two groups.

Group and group $\mathrm{x}$ emotion interaction

\begin{tabular}{lllll}
\hline Subjects & Group effect & $\begin{array}{l}\text { Marginal } \\
\text { effect }\end{array}$ & SE & $\boldsymbol{p}$ \\
\hline Controls & & 0.61 & 0.14 & $\mathbf{0 . 0 0 1}$ \\
ASD & & -0.58 & 0.13 & $\mathbf{0 . 0 0 1}$ \\
\hline \multirow{2}{*}{ Controls } & Group emotion interaction & & & \\
& Positive cognitive & 0.78 & 0.19 & $\mathbf{0 . 0 0 1}$ \\
& Negative cognitive & 0.88 & 0.19 & $\mathbf{0 . 0 0 1}$ \\
& Positive affective & 0.09 & 0.29 & 0.75 \\
& Negative affective & 0.69 & 0.21 & $\mathbf{0 . 0 0 1}$ \\
& Positive cognitive & -0.72 & 0.18 & $\mathbf{0 . 0 0 1}$ \\
& Negative cognitive & -0.72 & 0.19 & $\mathbf{0 . 0 0 1}$ \\
& Positive affective & -0.18 & 0.28 & 0.52 \\
& Negative affective & -0.69 & 0.21 & $\mathbf{0 . 0 0 1}$ \\
\hline
\end{tabular}

The significant results are highlighted by bold numbers.

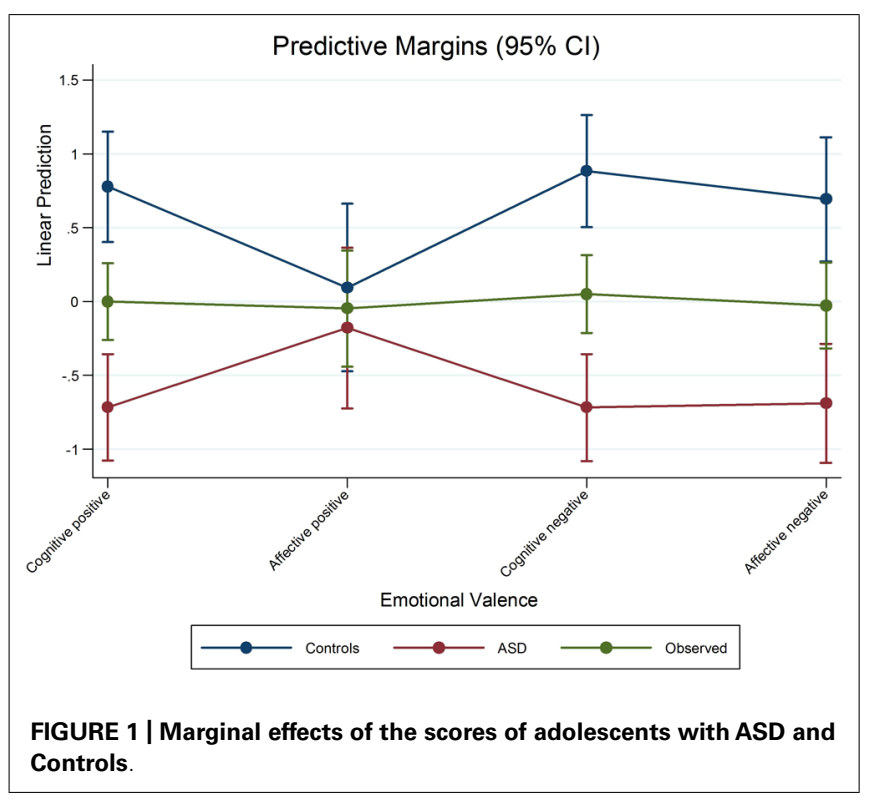

to normal controls, using a variety of assessment instruments, both paper and pencil and a computerized task. Our data show that adolescents with ASD have a deficit in the cognitive empathy dimension, but do not differ from controls in the affective empathy dimension when other people express emotions with positive valence. Their difficulty in empathizing with the emotional experience of other people is linked to sharing of emotions with negative valence.

Specifically, the results obtained in the paper and pencil measures that investigate mentalizing abilities reveal that the adolescents with ASD hardly interpret other people's mental states (First-order false belief and Advanced ToM Task) compared to controls. The ASD group also have trouble understanding the meaning of what other people are saying and doing, and they typically struggle to take the other person's perspective (BES-cognitive subscale).

The evaluation of mentalizing ability through false belief tasks is a key element in investigating the mentalizing skills in individuals with ASD. Therefore, these data confirm that ToM is a core deficit in ASD (Fletcher-Watson etal., 2014; Lai et al., 2014), which links both to precursor skills, such as joint attention and emotion recognition, and to subsequent abilities such as creating friendship and social inclusion. Instead, regarding the sharing experience measures (involving affective empathy, shared self-other representations and emotional contagion), adolescents with ASD were able to empathize with the emotional experience of other people when the latter expressed emotion with a positive valence. In contrast, they showed a deficit in sharing negative emotions. Moreover, the ASD group were unable to share other people's emotions by observing their ocular region (Eyes Task).

The results obtained in paper and pencil measures were confirmed by the computerized empathy task (MET, Dziobek et al., 2008).

The analysis of affective and cognitive empathy measures evaluated through the MET, showed significant differences between 
the adolescents with ASD and the control group in the cognitive empathy dimension both when they had to understand and recognize positive and negative emotions.

The cognitive empathic deficits of individuals with ASD could be due to a marked deficit in the ability to understand and explain the mental/emotional states of other people (Jones et al., 2010; Hirvela and Helkama, 2011; Samson et al., 2012; Schwenck et al., 2012; Lockwood et al., 2013).

As far as affective empathy is concerned, the ASD group do not show difficulties in the degree of empathic concern when the emotion is positive, whereas the difficulty is present when observing emotional images with negative valence. The adolescents with ASD feel aroused and involved when others experience positive emotions like the healthy subjects do. Therefore, our results obtained in both measures (paper and pencil and the computerized task) suggest that the ASD subjects showed a difficulty in cognitively identifying the mental state of other people, regardless of the different emotions to which they had to respond; on the other hand, the deficit in affective empathy is linked to emotional valence.

Several studies suggest that the processing of negative emotions is most difficult for individuals with autism (Howard et al., 2000; Ashwin et al., 2006; Corden et al., 2008; Wallace et al., 2008; Humphreys et al., 2013). The role of emotion in autism is still being debated. Ashwin et al. (2006) consider the difficulty of processing negative emotions in subjects with ASD to be linked to an atypical function and structure of the amygdala. In their study people with ASD were less accurate on the emotion recognition task compared to controls, but only for the negative basic emotions. This was discussed in the light of similar findings from people with damage to the amygdala. Based on our results, we assume that the impairment of experience sharing or affective empathy in adolescents with ASD is linked to their poor shared self-other representations of negative emotions.

Blair (2008) has proposed that one of the key processes underpinning functional affective empathy is the recognition of other people's distress cues (i.e., fear and sadness). Past studies (Howard et al., 2000; Blair, 2008) have shown that children and adolescents with psychopathic tendencies have difficulties in recognizing negative facial and vocal expressions. Thus, it is not possible to speak of impairment of the affective empathy dimension in adolescents with ASD without considering the type of emotion to which the subject responds. Emotional contagion for negative emotions of other people (like sadness, distress, suffering, anger) is important for adaptive social behavior. The lack of sharing experience when other people have negative emotions, leads to a failure of appropriate empathic behavior in adolescents with ASD.

Our results are important for the development of rehabilitation interventions that help these individuals to improve their social skills.

These results are in agreement with recent literature (Jones et al., 2010; Baron-Cohen, 2011; Cox et al., 2012; Schwenck et al., 2012; Lockwood et al., 2013). In particular, Baron-Cohen (2011) shows that cognitive empathy is impaired but affective empathy is not, in individuals with autism. On the contrary, in other psychological conditions, such as psychopathic personality disorder (borderline personality disorder, narcissism, psychopathy), an intact cognitive empathy and impaired affective empathy are present (Baron-Cohen, 2011). The lack of affective empathy, but not of cognitive empathy, seems be an important factor to promote violent and aggressive behaviors.

In conclusion, empathy is a multidimensional construct and requires three abilities: first, the recognition of emotions in oneself and other people via facial expressions, shown by the gaze or behavior; second, the sharing of emotional states with others, i.e., the ability to experience similar emotions to other people while being conscious that this is a simulation of the emotional feeling and it is not one's own emotion (Derntl et al., 2010) and finally to take the perspective of another person, though the distinction between one's self and other people remains intact (Decety and Jackson, 2004). For this reason, it is important to use more instruments that allow us to capture all aspects of empathy. Our approach enabled a more detailed analysis of these empathic competencies, also considering the role of emotions in the empathic construct. We believe that this dissociation in cognitive and affective empathy is of importance for several psychiatric conditions which show the empathic ability impairment, such as autism spectrum disorder but also schizophrenia (Fujino et al., 2014) or post-traumatic stress disorder (Mazza et al., 2013). Replication with a larger sample of ASD subjects will be necessary to confirm the present findings.

\section{AUTHOR CONTRIBUTIONS}

Monica Mazza and Marco Valenti designed research; Maria C. Pino, Melania Mariano, and Daniela Tempesta collected data; Monica Mazza, Francesco Masedu, and Domenico De Berardis analyzed the data; Monica Mazza, Maria C. Pino, Michele Ferrara, and Marco Valenti wrote paper.

\section{REFERENCES}

Albiero, P., Matricardi, G., Speltri, D., and Toso, D. (2009). The assessment of empathy in adolescence: a contribution to the Italian validation of the "Basic Empathy Scale.” J. Adolesc. 32, 393-408. doi: 10.1016/j.adolescence.2008.01.001

American Psychiatric Association. (2000). Diagnostic and Statistical Manual of Mental Disorders: DSM-IV-TR, 4th Edn. Washington, DC: American Psychiatric Press.

American Psychiatric Association. (2013). Diagnostic and Statistical Manual of Mental Disorders: DSM-V, 5th edn. Arlington, VA: American Psychiatric Publishing.

Ashwin, C., Chapman, E., Colle, L., and Baron-Cohen, S. (2006). Impaired recognition of negative basic emotions in autism: a test of the amygdala theory. Soc. Neurosci. 1, 349-363. doi: 10.1080/17470910601040772

Baron-Cohen, S. (2011). Zero Degrees of Empathy: A New Theory of Human Cruelty. London: Penguin/Allen Lane.

Baron-Cohen, S., Wheelwright, S., Hill, J., Raste, Y., and Plumb, I. (2001). The "reading the mind in the eyes" test revised version: a study with normal adults, and adults with Asperger syndrome or high-functioning autism. J. Child Psychol. Psychiatry. 42, 241-251. doi: 10.1111/1469-7610.00715

Blair, R. J. (2008). Fine cuts of empathy and the amygdala: dissociable deficits in psychopathy and autism. Q. J. Exp. Psychol. (Hove) 61, 157-170. doi: $10.1080 / 17470210701508855$

Blair, R. J., and Cipolotti, L. (2000). Impaired social response reversal. A case of "acquired sociopathy". Brain 123, 1122-1141. doi: 10.1093/brain/123.6.1122

Corden, B., Chilvers, R., and Skuse, D. (2008). Emotional modulation of perception in Asperger's syndrome. J. Autism Dev. Disord. 38, 1072-1080. doi: 10.1007/s10803-007-0485-y

Cox, C. L., Uddin, L. Q., Di Martino, A., Castellanos, F. X., Milham, M. P., and Kelly, C. (2012). The balance between feeling and knowing: affective and cognitive empathy are reflected in the brain's intrinsic functional dynamics. Soc. Cogn. Affect. Neurosci. 7, 727-737. doi: 10.1093/scan/nsr051 
Davis, M. H. (1980). A multidimensional approach to individual differences in empathy. JSAS Catalog Select. Doc. Psychol. 10, 85.

Decety, J., and Jackson, P. L. (2004). The functional architecture of human empathy. Behav. Cogn. Neurosci. Rev. 3, 71-100. doi: 10.1177/1534582304267187

Decety, J., and Meyer, M. (2008). From emotion resonance to empathic understanding: a social developmental neuroscience account. Dev. Psychopathol. 20, 1053-1080. doi: 10.1017/S0954579408000503

Derntl, B., Finkelmeyer, A., Eickhoff, S., Kellermann, T., Falkenberg, D. I., Schneider, F., et al. (2010). Multidimensional assessment of empathic abilities: neural correlates and gender differences. Psychoneuroendocrinology 35, 67-82. doi: 10.1016/j.psyneuen.2009.10.006

Dziobek, I., Preissler, S., Grozdanovic, Z., Heuser, I., Heekeren, H. R., and Roepke, S. (2011). Neuronal correlates of altered empathy, and social cognition in borderline personality disorder. Neuroimage 15, 539-548. doi: 10.1016/j.neuroimage.2011.05.005

Dziobek, I., Rogers, K., Fleck, S., Bahnemann, M., Heekeren, H. R., Wolf, O. T., et al. (2008). Dissociation of cognitive and emotional empathy in adults with Asperger syndrome using the Multifaceted Empathy Test (MET). J. Autism Dev. Disord. 38, 464-473. doi: 10.1007/s10803-007-0486-x

Fletcher-Watson, S., McConnell, F., Manola, E., and McConachie, H. (2014) Interventions based on the Theory of Mind cognitive model for autism spectrum disorder (ASD). Cochrane Database Syst. Rev. 3:CD008785. doi: 10.1002/14651858.CD008785.pub2

Frith, U., and Frith, C. D. (2003). Development and neurophysiology of mentalizing. Philos. Trans. R. Soc. Lond. B Biol. Sci. 29, 459-473. doi: 10.1098/rstb.2002.1218

Frith, U., and Happé, F. (1994). Autism: beyond "theory of mind.” Cognition 50, 115-132. doi: 10.1016/0010-0277(94)90024-8

Fujino, J., Takahashi, H., Miyata, J., Sugihara, G., Kubota, M., Sasamoto, A., et al. (2014). Impaired empathic abilities and reduced white matter integrity in schizophrenia. Prog. Neuropsychopharmacol. Biol. Psychiatry 48, 117-123. doi: 10.1016/j.pnpbp.2013.09.018

Gaigg, S. B. (2012). The interplay between emotion and cognition in autism spectrum disorder: implications for developmental theory. Front. Integr. Neurosci. 6:113. doi: $10.3389 /$ fnint.2012.00113

Hadjikhani, N., Zürcher, N. R., Rogier, O., Hippolyte, L., Lemonnier, E., Ruest, T., et al. (2014). Emotional contagion for pain is intact in autism spectrum disorders. Transl. Psychiatry 14, 4-343. doi: 10.1038/tp.2013.113

Happé, F. (1994). An advanced test of theory of mind: understanding of story characters' thoughts and feelings by able autistics, mentally handicapped and normal children and adults. J. Autism Dev. Disord. 24, 129-154. doi: 10.1007/BF02172093

Hirvela, S., and Helkama, K. (2011). Empathy, values, morality and Asperger's syndrome. Scand. J. Psychol. 52, 560-572. doi: 10.1111/j.1467-9450.2011.00913.x

Howard, M. A., Cowell, P. E., Boucher, J., Broks, P., Mayes, A., Farrant, A., et al. (2000). Convergent neuroanatomical and behavioural evidence of an amygdala hypothesis of autism. Neuroreport 11, 2931-2935. doi: 10.1097/00001756200009110-00020

Humphreys, T., Polick, A. S., Howk, L. L., Thaxton, J. R., and Ivancic, A. P. (2013) An evaluation of repeating the discriminative stimulus when using least-to-most prompting to teach intraverbal behavior to children with autism. J. Appl. Behav. Anal. 46, 534-538. doi: 10.1002/jaba.43

Jolliffe, D., and Farrington, D. P. (2006). Development and validation of the Basic Empathy Scale. J. Adolesc. 29, 589-611. doi: 10.1016/j.adolescence.2005.08.010

Jones, A. P., Happé, F. G. E., Gilbert, F., Burnett, S., and Viding, E. (2010). Feeling, caring, knowing: different types of empathy deficit in boys with psychopathic tendencies and autism spectrum disorder. J. Child Psychol. Psychiatry 11, 11881197. doi: 10.1111/j.1469-7610.2010.02280.x

Lai, M. C., Lombardo, M. V., and Baron-Cohen S. (2014). Autism. Lancet 8, 896-910. doi: 10.1016/S0140-6736(13)61539-1

Lang, P. J. (1980) "Behavioral treatment and bio-behavioral assessment: computer applications," in Technology in Mental Health and Delivery Systems, eds J. B. Sidowski, J. H. Johnson, and T. A. Williams (Norwood, MA: Ablex), 119-137.

Lockwood, P. L., Bird, G., Bridge, M., and Viding, E. (2013). Dissecting empathy: high levels of psychopathic and autistic traits are characterized by difficulties in different social information processing domains. Front. Hum. Neurosci. 7:760 doi: 10.3389/fnhum.2013.00760

Lord, C., Rutter, M., DiLavore, P. C., Risi, S., Gotham, K., and Bishop, S. L., (2012). Autism Diagnostic Observation Schedule (ADOS-2): Manual, 2nd Edn. Los Angeles, CA: Western Psychological Services.
Mazza, M., Costagliola, C., Di Michele, V., Magliani, V., Pollice, R., Ricci, A., et al. (2007). Deficit of social cognition in subjects with surgically treated frontal lobe lesions and in subjects affected by schizophrenia. Eur. Arch. Psychiatry Clin. Neurosci. 257, 12-22. doi: 10.1007/s00406-006-0676-0

Mazza, M., Tempesta, D., Pino, M. C., Catalucci, A., Gallucci, M., and Ferrara, M. (2013). Regional cerebral changes and functional connectivity during the observation of negative emotional stimuli in subjects with posttraumatic stress disorder. Eur. Arch. Psychiatry Clin. Neurosci. 263, 575-583. doi: 10.1007/s00406013-0394-3

Ponnet, K. S., Roeyers, H., Buysse, A., De Clercq, A., and Van der Heyden, E. (2004). Advanced mind-reading in adults with Asperger syndrome. Autism 8, 249-266. doi: $10.1177 / 1362361304045214$

Rogers, K., Dziobek, I., Hassenstab, J., Wolf, O. T., and Convit, A. (2007). Who cares? Revisiting empathy in Asperger syndrome. J. Autism Dev. Disord. 37, 709-715. doi: 10.1007/s10803-006-0197-8

Rowe, A. D., Bullock, P. R., and Polkey, C. E. (2001). “Theory of mind” impairments and their relationship to executive functioning following frontal lobe excisions. Brain 124, 600-616. doi: 10.1093/brain/124.3.600

Samson, A. C., Huber, O., and Gross, J. J. (2012). Emotion regulation in Asperger's syndrome and high-functioning autism. Emotion 12, 659-665. doi: $10.1037 / \mathrm{a} 0027975$

Schwenck, C., Mergenthaler, J., Keller, K., Zech, J., Salehi, S., Taurines, R., et al. (2012). Empathy in children with autism and conduct disorder: group-specific profiles and developmental aspects. J. Child Psychol. Psychiatry 53, 651-659. doi: 10.1111/j.1469-7610.2011.02499.x

Shamay-Tsoory, S. G. (2011). The neural bases for empathy. Neuroscientist 17, 18-24. doi: 10.1177/1073858410379268

Shamay-Tsoory, S. G., Aharon-Peretz, J., and Perry, D. (2009). Two systems for empathy: a double dissociation between emotional and cognitive empathy in inferior frontal gyrus versus ventromedial prefrontal lesions. Brain 132, 617-627. doi: 10.1093/brain/awn279

Shamay-Tsoory, S. G., Tomer, R., Yaniv, S., and Aharon-Peretz, J. (2002). Empathy deficits in Asperger syndrome: a cognitive profile. Neurocase 8, 245-252. doi: 10.1093/neucas/8.3.245

Singer, T. (2006). The neuronal basis and ontogeny of empathy and mind reading: review of literature and implications for future research. Neurosci. Biobehav. Rev. 30, 855-863. doi: 10.1016/j.neubiorev.2006.06.011

Sucksmith, E., Allison, C., Baron-Cohen, S., Chakrabarti, B., and Hoekstra, R. A. (2013). Empathy and emotion recognition in people with autism, first-degree relatives, and controls. Neuropsychologia 51, 98-105. doi: 10.1016/j.neuropsychologia.2012.11.013

Vellante, M., Baron-Cohen, S., Melis, M., Marrone, M., Petretto, D. R., Masala, C., et al. (2013). The "Reading the Mind in the Eyes" test: systematic review of psychometric properties and a validation study in Italy. Cogn. Neuropsychiatry 18, 326-354 doi: 10.1080/13546805.2012.721728

Wallace, S., Coleman, M., and Bailey, A. (2008). Face and object processing in autism spectrum disorders. Autism Res. 1, 43-51. doi: 10.1002/aur.7

Wilkinson, L. (2012). DSM-5: Rethinking Asperger's Disorder. Autism 2:e113. doi: $10.4172 / 2165-7890.1000 \mathrm{e} 113$

Zaki, J., and Ochsner, K. N. (2012). The neuroscience of empathy: progress, pitfalls and promise. Nat. Neurosci. 15, 675-680. doi: 10.1038/nn.3085

Conflict of Interest Statement: The authors declare that the research was conducted in the absence of any commercial or financial relationships that could be construed as a potential conflict of interest.

Received: 06 June 2014; accepted: 17 September 2014; published online: 07 October 2014.

Citation: Mazza M, Pino MC, Mariano M, Tempesta D, Ferrara M, De Berardis $D$, Masedu F and Valenti $M$ (2014) Affective and cognitive empathy in adolescents with autism spectrum disorder. Front. Hum. Neurosci. 8:791. doi: 10.3389/fnhum.2014.00791

This article was submitted to the journal Frontiers in Human Neuroscience.

Copyright (C) 2014 Mazza, Pino, Mariano, Tempesta, Ferrara, De Berardis, Masedu and Valenti. This is an open-access article distributed under the terms of the Creative Commons Attribution License (CC BY). The use, distribution or reproduction in other forums is permitted, provided the original author(s) or licensor are credited and that the original publication in this journal is cited, in accordance with accepted academic practice. No use, distribution or reproduction is permitted which does not comply with these terms. 